\title{
Efficient Bounded Distance Decoders for Barnes-Wall Lattices*
}

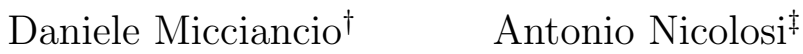

April 30, 2008

\begin{abstract}
We describe a new family of parallelizable bounded distance decoding algorithms for the Barnes-Wall lattices, and analyze their decoding complexity. The algorithms are parameterized by the number $p=4^{k} \leq N^{2}$ of available processors, work for Barnes-Wall lattices in arbitrary dimension $N=2^{n}$, correct any error up to squared unique decoding radius $d_{\min }^{2} / 4$, and run in worst-case time $O\left(N \log ^{2} N / \sqrt{p}\right)$. Depending on the value of the parameter $p$, this yields efficient decoding algorithms ranging from a fast sequential algorithm with quasi-linear decoding complexity $O\left(N \log ^{2} N\right)$, to a fully parallel decoding circuit with polylogarithmic depth $O\left(\log ^{2} N\right)$ and polynomially many arithmetic gates.
\end{abstract}

\section{Introduction: Barnes-Wall Lattices}

Barnes-Wall lattices are an infinite sequence of full-rank lattices defined for every dimension $N$ that is a power of 2. For their elegant simplicity and relevance to practical applications, Barnes-Wall lattices have been the subject of extensive investigations in coding theory $[2,1,8,9,3,4,12,11]$ and mathematics $[7,6]$. We use the definition of Barnes-Wall lattice $\mathrm{BW}^{n}$ as $N=2^{n}$ dimensional lattices over the Gaussian integers $\mathbb{G}=\mathbb{Z}+i \mathbb{Z}$.

Definition 1. For any positive integer $n, B W^{n}$ is the $N=2^{n}$ dimensional lattice over $\mathbb{G}$ generated by the rows of the $n$-fold Kronecker product

$$
B W^{n}=\left[\begin{array}{ll}
1 & 1 \\
0 & \phi
\end{array}\right]^{\otimes n}
$$

where $\phi=1+i$ is the prime of least squared norm in $\mathbb{G}$, i.e., the $N \times N$ matrix defined by the recurrence

$$
B W^{n}=\left[\begin{array}{cc}
B W^{n-1} & B W^{n-1} \\
\mathbf{O} & \phi \cdot B W^{n-1}
\end{array}\right]
$$

with initial condition $B W^{0}=[1]$.

\footnotetext{
${ }^{*}$ To appear in the proceedings of the IEEE International Symposium on Information Theory - ISIT 2008.

${ }^{\dagger}$ Computer Science \& Engineering Department, University of California, San Diego, daniele@cs.ucsd.edu

${ }^{\ddagger}$ Computer Science Department, Stevens Institute of Technology. nicolosi@cs.stevens.edu
} 


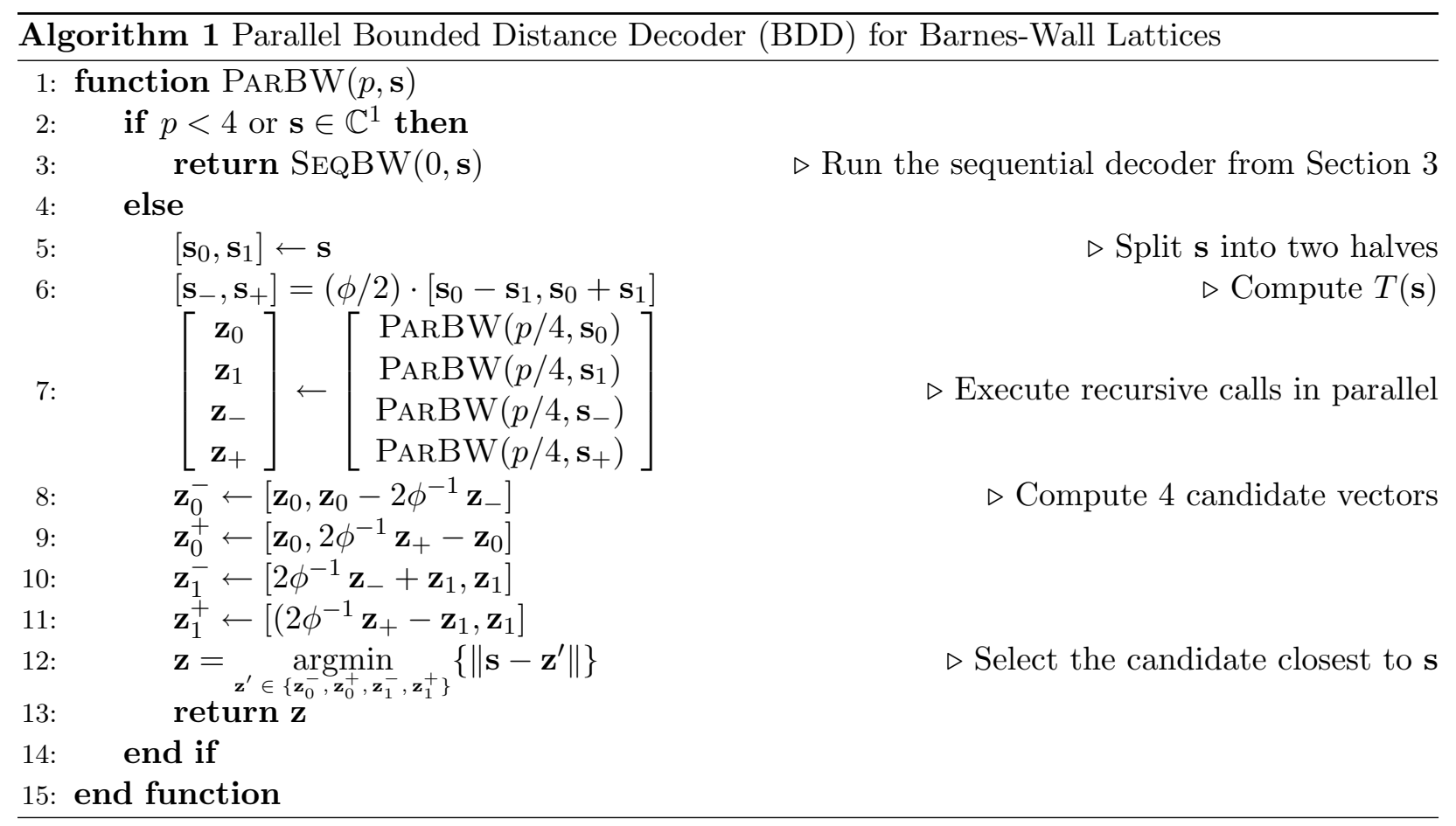

Equivalently, $\mathrm{BW}^{n}$ can be defined as a $2 N=2^{n+1}$ dimensional lattice over the integers in the obvious way, but complex numbers make our definitions and algorithms easier to describe. It immediately follows from the definition that $\mathrm{BW}^{0}=\mathbb{G}$ is the 1-dimensional lattice of all Gaussian integers, and

$$
\mathrm{BW}^{n+1}=\left\{[\mathbf{u}, \mathbf{u}+\phi \mathbf{v}]: \mathbf{u}, \mathbf{v} \in \mathrm{BW}^{n}\right\}, \quad \text { for } n \geq 0 .
$$

The Barnes-Wall lattices have minimum squared distance $d_{\min }^{2}\left(\mathrm{BW}^{n}\right)=N$, volume $V\left(\mathrm{BW}^{n}\right)=$ $2^{n 2^{n-1}}=\sqrt{N^{N}}$, and nominal coding gain $\gamma_{c}\left(\mathrm{BW}^{n}\right)=2^{n / 2}=\sqrt{N}$.

Although much effort has been put in the design of efficient decoding algorithms for BarnesWall lattices in specific low dimensions (like $\mathrm{BW}^{2}$ and $\mathrm{BW}^{3},[8,12]$ ), not much is known about the asymptotic complexity of decoding $\mathrm{BW}^{n}$. For arbitrary $n$, the only decoding algorithms explicitly discussed in the literature are those based on the four-section, $2^{N / 2}$-state trellis realization of $\mathrm{BW}^{n}$ (cf. e.g. [3]), which accomplish maximum likelihood decoding but have exponential (in $N$ ) complexity.

In this paper, we give a family of efficient (polynomial time) algorithms to solve the bounded distance decoding problem for Barnes-Wall lattices: given a vector $\mathbf{s} \in \mathbb{C}^{N}$ within squared distance $d_{\min }^{2} / 4=N / 4$ from some lattice point $\mathbf{z}$ in $\mathrm{BW}^{n}$, find $\mathbf{z}$. Our family of algorithms is parameterized by an integer $p=4^{k}$, ranging from $1=4^{0}$ to $N^{2}=4^{n}$, that represents the number of available processors. The (parallel) running time of the algorithm (measured in terms of arithmetic operations) is $O\left(N \log ^{2} N / \sqrt{p}\right)$. All arithmetic is performed using at most $n=\log _{2} N$ bits of precisions, beyond the precision used to represent the target vector $\mathbf{s} \in \mathbb{C}^{N}$.

\section{The Parallel Bounded Distance Decoder}

The algorithm is based on the following easily verifiable observations: 


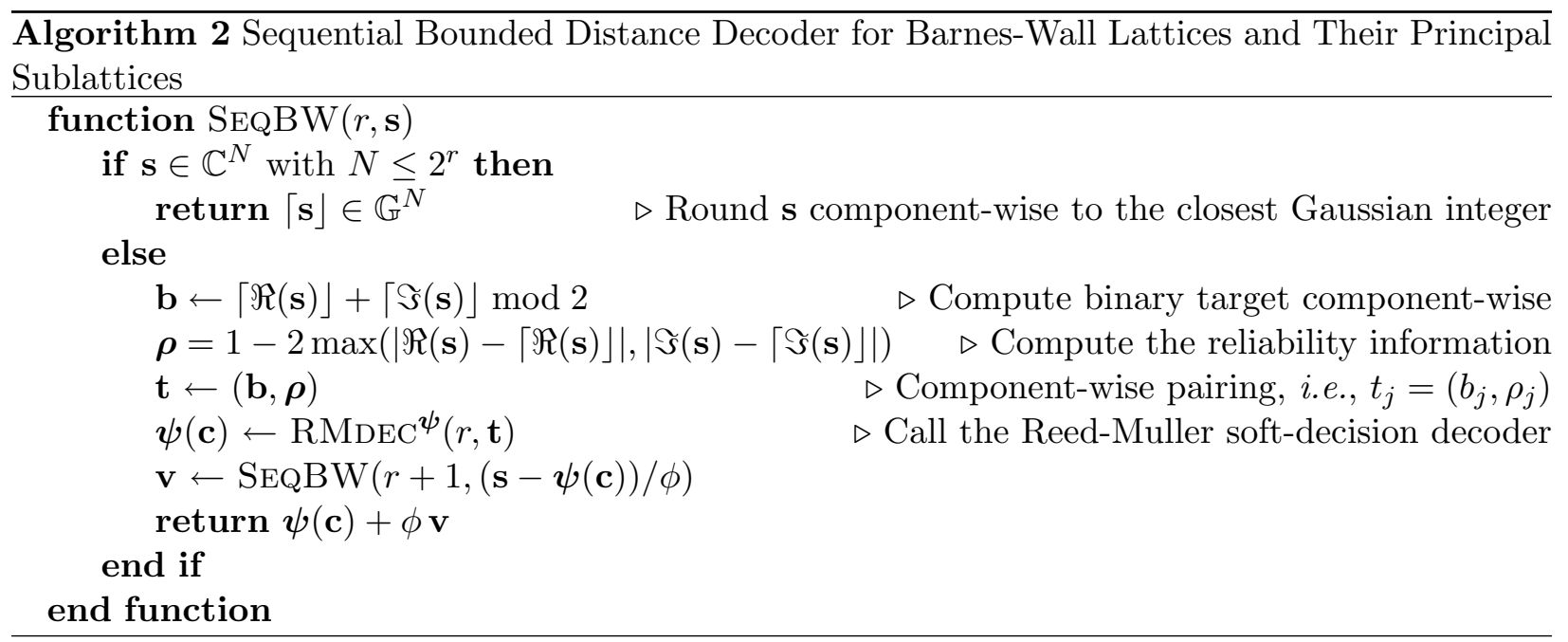

- If $\left[\mathbf{z}_{0}, \mathbf{z}_{1}\right] \in \mathrm{BW}^{n+1}$, then $\mathbf{z}_{0}, \mathbf{z}_{1} \in \mathrm{BW}^{n}$.

- $\left\|\left[\mathbf{s}_{0}, \mathbf{s}_{1}\right]\right\|^{2}=\left\|\mathbf{s}_{0}\right\|^{2}+\left\|\mathbf{s}_{1}\right\|^{2}$, so if $\left[\mathbf{s}_{0}, \mathbf{s}_{1}\right]$ is within the squared unique decoding radius of $\mathrm{BW}^{n+1}$ $\left(d_{\text {min }}^{2}\left(\mathrm{BW}^{n+1}\right) / 4=N / 2\right)$, then at least one among $\mathbf{s}_{0}$ and $\mathbf{s}_{1}$ is within the squared unique decoding radius $d_{\text {min }}^{2}\left(\mathrm{BW}^{n}\right) / 4=N / 4$ of $\mathrm{BW}^{n}$.

- The function:

$$
T:\left[\mathbf{z}_{0}, \mathbf{z}_{1}\right] \mapsto(\phi / 2) \cdot\left[\mathbf{z}_{0}-\mathbf{z}_{1}, \mathbf{z}_{0}+\mathbf{z}_{1}\right]
$$

is an automorphism of $\mathrm{BW}^{n}$, i.e., a distance preserving linear transformation that maps $\mathrm{BW}^{n}$ to itself.

- The vectors $\mathbf{z}_{0}$ and $\mathbf{z}_{1}$ can be recovered from any of the following pairs: $\left(\mathbf{z}_{0}, \mathbf{z}_{-}\right),\left(\mathbf{z}_{0}, \mathbf{z}_{+}\right)$, $\left(\mathbf{z}_{1}, \mathbf{z}_{-}\right),\left(\mathbf{z}_{1}, \mathbf{z}_{+}\right)$, where $\left[\mathbf{z}_{-}, \mathbf{z}_{+}\right]=T\left(\left[\mathbf{z}_{0}, \mathbf{z}_{1}\right]\right)$.

These observations translate pretty much directly into Algorithm 1, reported above.

Theorem 1. For any $N=2^{n}, 1 \leq p \leq N^{2}$, and $\mathbf{s} \in \mathbb{C}^{N}$ such that dist ${ }^{2}\left(\mathbf{s}, B W^{n}\right)<N / 4$, Algorithm 1 computes the (unique) lattice vector $z \in B W^{n}$ within squared distance $N / 4$ from the target vector $\mathbf{s}$.

Proof. The proof easily follows from the previous observations and from the correctness of the sequential decoder SEQBW given in Section 3. Let $\left[\tilde{\mathbf{z}}_{0}, \tilde{\mathbf{z}}_{1}\right]$ be the lattice point within squared distance $N / 4$ from the target $\left[\mathbf{s}_{0}, \mathbf{s}_{1}\right]$. Since $T$ is an automorphism of $\mathrm{BW}^{n}$, also the target $\left[\mathbf{s}_{-}, \mathbf{s}_{+}\right]=$ $T\left(\left[\mathbf{s}_{0}, \mathbf{s}_{1}\right]\right)$ is within squared distance from $\mathrm{BW}^{n}$, and the closest lattice point to it is $\left[\tilde{\mathbf{z}}_{-}, \tilde{\mathbf{z}}_{+}\right]=$ $T\left(\left[\tilde{\mathbf{z}}_{0}, \tilde{\mathbf{z}}_{1}\right]\right)$.

The algorithm recursively computes four $N / 2$-dimensional vectors $\mathbf{z}_{\star}$ (for $\star \in\{0,1,+,-\}$ ) with the property that if $\mathbf{s}_{\star}$ is within squared distance $N / 2$ from $\mathrm{BW}^{n-1}$, then $\mathbf{z}_{\star}=\tilde{\mathbf{z}}_{\star}$. Next, for each $b \in\{0,1\}$ and $s \in\{-,+\}$, the algorithm computes a candidate vector $\mathbf{z}_{b}^{s}$ from $\left[\mathbf{z}_{b}, \mathbf{z}_{s}\right]$ by inverting the linear transformation that maps $\left[\tilde{\mathbf{z}}_{0}, \tilde{\mathbf{z}}_{1}\right]$ to $\left[\tilde{\mathbf{z}}_{b}, \tilde{\mathbf{z}}_{s}\right]$.

Since at least one vector from each pair $\left(\mathbf{s}_{0}, \mathbf{s}_{1}\right)$ and $\left(\mathbf{s}_{-}, \mathbf{s}_{+}\right)$is within the unique decoding radius from the lattice, the algorithm correctly recovers $\left[\mathbf{z}_{b}, \mathbf{z}_{s}\right]=\left[\tilde{\mathbf{z}}_{b}, \tilde{\mathbf{z}}_{s}\right]$ for some $b \in\{0,1\}$ and $s \in\{-,+\}$, and $\mathbf{z}_{b}^{s}=\left[\tilde{\mathbf{z}}_{0}, \tilde{\mathbf{z}}_{1}\right]$. Selecting the vector among $\mathbf{z}_{0}^{-}, \mathbf{z}_{0}^{+}, \mathbf{z}_{1}^{-}, \mathbf{z}_{1}^{+}$closest to the target 
correctly identifies $\left[\tilde{\mathbf{z}}_{0}, \tilde{\mathbf{z}}_{1}\right]$ because $\left[\tilde{\mathbf{z}}_{0}, \tilde{\mathbf{z}}_{1}\right]$ is the only lattice vector within the unique decoding radius from the lattice.

Theorem 2. For any $N=2^{n}, 1 \leq p \leq N^{2}$, and $\mathbf{s} \in \mathbb{C}^{N}$, the execution of Algorithm 1 on $p$ processors terminates after $O\left(N \log ^{2} N / \sqrt{p}\right)$ steps on each processor.

Proof. Performing steps $5-6$ and 8-11 of Algorithm 1 clearly takes $O(\max \{1, N / p\})$ parallel time. Computing the distance between the four candidates and the target vector (step 12) entails the evaluation of summations with $N$ terms, each requiring $\log N$ sequential rounds, and overall $O(\log N+N / p)$ parallel time. As a result, the running time $T_{1}(p, N)$ of Algorithm 1 on $p$ processors for inputs of size $N$ satisfies the recurrence:

$$
T_{1}(p, N)=\left\{\begin{array}{l}
T_{2}(0, N) \quad \text { if } p<4 \text { or } N=1 \\
O(\log N+N / p)+T_{1}(p / 4, N / 2) \quad \text { o/w }
\end{array}\right.
$$

where $T_{2}(r, N)=(\log N-r)(N \log N)$ is the running time of the sequential decoder SEQBW $(c f$. Section 3 of Algorithm 1). When $p=N^{2}=4^{n}$, the recursion unfolds exactly $n$ times and terminates with $p=N=1$, yielding $T_{1}\left(N^{2}, N\right)=O\left(\log ^{2} N\right)$. When $p=4^{k}, k<n$, the running time is dominated by the sequential decoding (cf. step 3) of a vector of residual length $N / 2^{k}=N / \sqrt{p}$, yielding $T_{1}(p, N)=O\left(T_{2}(0, N / \sqrt{p})\right)=O\left(N \log ^{2} N / \sqrt{p}\right)$.

\section{The Sequential Bounded Distance Decoder}

In this section we present a sequential algorithm for decoding Barnes-Wall lattices up to their squared unique decoding radius. The algorithm is based on the multilevel construction $[4,1]$ of Barnes-Wall lattices from Reed-Muller codes, and employs the soft decision decoder of $[10,5]$.

Definition 2. For any $r \leq n$, the Reed-Muller code $R M_{r}^{n}$ is the $N=2^{n}$ dimensional binary linear code defined by

$$
R M_{r}^{n}=\left\{\left[p(\mathbf{x}): \mathbf{x} \in \mathbb{F}_{2}^{n}\right]: p \in \mathbb{F}_{2}[\mathbf{x}], \operatorname{deg}(p) \leq r\right\} .
$$

It follows from the definition that $\mathrm{RM}_{r}^{n}$ satisfies $\mathrm{RM}_{0}^{n}=\{\mathbf{0}, \mathbf{1}\}, \mathrm{RM}_{n}^{n}=\mathbb{F}_{2}^{N}$ and, for $0<r<n$, $\mathrm{RM}_{r}^{n}=\left\{[\mathbf{u}, \mathbf{u} \oplus \mathbf{v}]: \mathbf{u} \in \mathrm{RM}_{r}^{n-1}, \mathbf{v} \in \mathrm{RM}_{r-1}^{n-1}\right\}$. The binary code $\mathrm{RM}_{r}^{n}$ has block length $N=2^{n}$, dimension $k=\sum_{s<r}\left(\begin{array}{l}n \\ s\end{array}\right)$ and minimum distance $d=2^{n-r}$.

Notice that Reed-Muller codewords are vectors in $\mathbb{F}_{2}^{N}$, but for the purposes of our decoding algorithms we need to interpret them as vectors in $\mathrm{BW}^{n} \subset \mathbb{G}^{N}$. This can be done via the following linear transformation $\psi: \mathbb{F}_{2}^{N} \rightarrow \mathbb{G}^{N}$ :

$$
\left\{\begin{array}{l}
\psi(\mathbf{0})=\mathbf{0} \\
\boldsymbol{\psi}(\mathbf{1})=\mathbf{1} \\
\boldsymbol{\psi}([\mathbf{u}, \mathbf{u} \oplus \mathbf{v}])=[\boldsymbol{\psi}(\mathbf{u}), \psi(\mathbf{u})+\psi(\mathbf{v})]
\end{array}\right.
$$

The relation between Barnes-Wall lattices and Reed-Muller codes can then be described as follows ( $c f$. also [3], Section IV.B): 
Theorem 3. Each lattice vector $\mathbf{v} \in B W^{n}$ can be uniquely expressed as

$$
\mathbf{v}=\sum_{r=0}^{n-1} \phi^{r} \boldsymbol{\psi}\left(\mathbf{c}_{r}\right)+\phi^{n} \mathbf{c}_{n}
$$

where $\mathbf{c}_{n} \in \mathbb{G}^{N}$ and $\mathbf{c}_{r} \in R M_{r}^{n}$ for $r=0, \ldots, n-1$.

For any $0 \leq r \leq n$, let

$$
\begin{array}{r}
\mathrm{BW}_{r}^{n}=\left\{\sum_{k=r}^{n-1} \phi^{k-r} \psi\left(\mathbf{c}_{k}\right)+\phi^{n-r} \mathbf{c}_{n} \quad:\right. \\
\left.\mathbf{c}_{k} \in \mathrm{RM}_{k}^{n}, \mathbf{c}_{n} \in \mathbb{G}^{2^{n}}\right\} .
\end{array}
$$

be the so-called principal sublattices of $\mathrm{BW}^{n}$ ( $c f$. [3], Section IV.B). In other words, $\mathrm{BW}_{r}^{n}$ is the set of all lattice vectors in $\mathrm{BW}^{n}$ such that $\mathbf{c}_{0}=\ldots=\mathbf{c}_{r-1}=\mathbf{0}$, scaled by a factor $\phi^{r}$. It is clear that each set $\mathrm{BW}_{r}^{n}$ is itself a lattice, i.e., it is closed under addition and subtraction.

Algorithm 2 above defines a sequential decoder $\operatorname{SeQBW}(r, \mathbf{s})$ for this family of lattices. When $r=0, \operatorname{SEQBW}(0, \mathbf{s})$ gives a decoder for $\mathrm{BW}_{0}^{n}=\mathrm{BW}^{n}$.

Theorem 4. For any $N=2^{n}, r \leq n$, and $\mathbf{s} \in \mathbb{C}^{N}$ such that dist ${ }^{2}\left(\mathbf{s}, B W_{r}^{n}\right)<N / 2^{r+2}$, Algorithm 2 computes the (unique) lattice vector $\mathbf{z} \in B W_{r}^{n}$ within squared distance $N / 2^{r+2}$ from $\mathbf{s}$.

In order to complete the description of the sequential decoding algorithm, we need to give a soft decision decoder for Reed-Muller codes in Euclidean space. Algorithm 3 is essentially the softdecision decoder of [10], with the following differences: 1) our algorithm uses additive 0,1 notation for vectors, whereas [10] represents codewords as vectors in $\left.\{-1,+1\}^{N} ; 2\right)$ we combine the softdecision decoding of the Reed-Muller code with the linear embedding $\psi: \mathrm{RM}_{r}^{n} \rightarrow \mathrm{BW}_{r}^{n}$. We remark that the image of $\{0,1\}^{N}$ under $\boldsymbol{\psi}$ is a subset of $\mathbb{Z}^{N}$. So, on input a vector $\mathbf{t} \in(\{0,1\} \times[0,1])^{N}$, Algorithm 3 outputs a vector $\operatorname{RMDEC}^{\psi}(r, \mathbf{t}) \in \mathbb{Z}^{N} \cap \mathrm{BW}_{r}^{n}$.

For any $N=2^{n}, 0 \leq r \leq n$, and $\mathbf{t} \in(\{0,1\} \times[0,1])^{N}$, the running time $T_{3}(r, N)$ of Algorithm 3 is described by the recurrence:

$$
T_{3}(r, N)=\left\{\begin{array}{l}
O(N) \quad \text { if } r=0 \text { or } N=2^{r} \\
O(N)+T_{3}(r-1, N / 2)+T_{3}(r, N / 2) \quad \mathrm{o} / \mathrm{w}
\end{array}\right.
$$

which is easily seen to satisfy:

$$
T_{3}(r, N)=O(N \log N) .
$$

Since Algorithm 2 essentially amounts to iterative decoding of length- $N$ Reed-Muller codewords of order ranging from $r$ to $(\log N-1)$, it follows that its running time grows asymptotically as:

$$
T_{2}(r, N)=O(\log N-r)(N \log N) .
$$

\section{Open Problems}

Our investigation on efficient bounded distance decoding for Barnes-Wall lattices brings up several questions and directions for future work: Is it possible to improve the efficiency of the BDD 


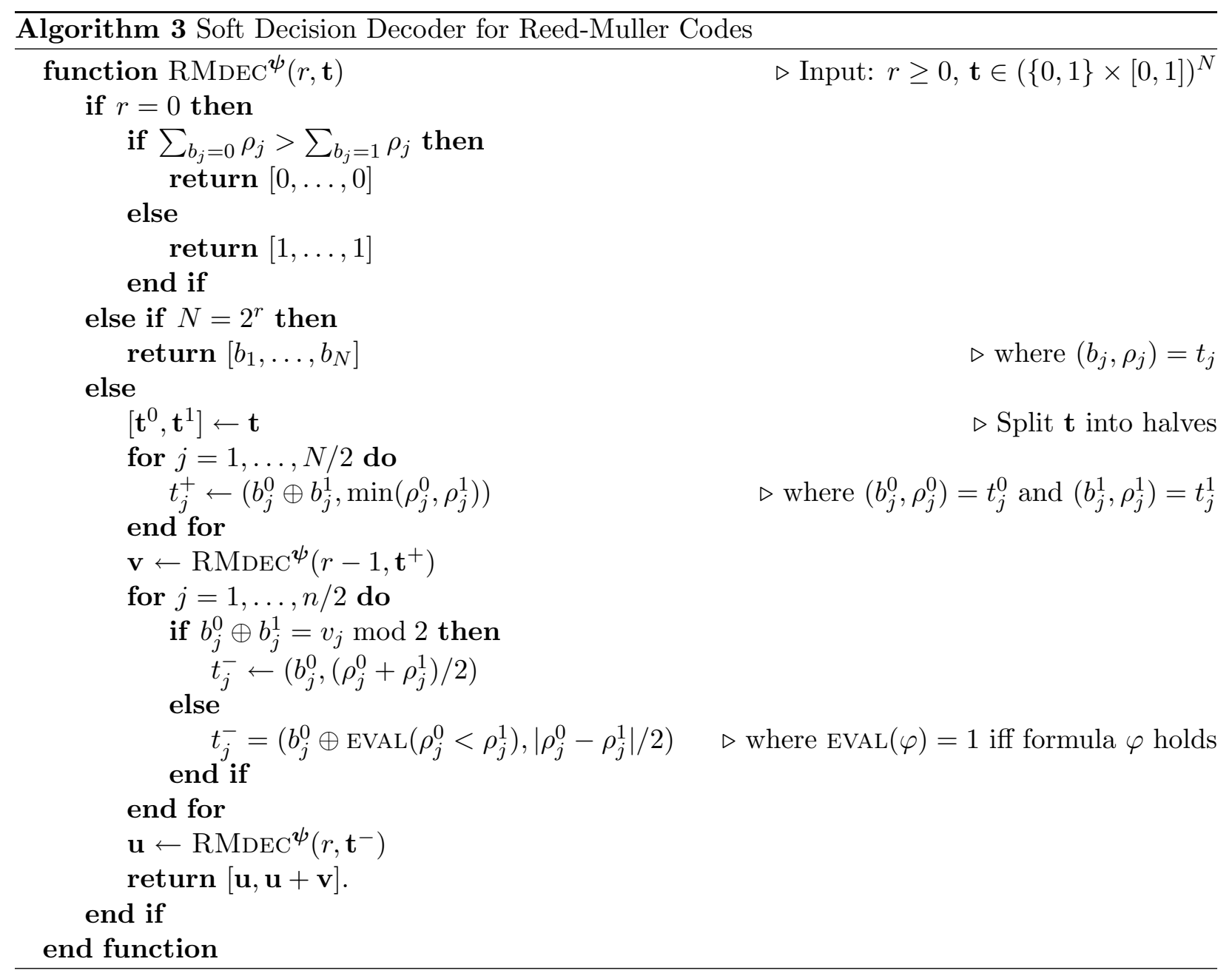

algorithm given in this paper? In particular, is it possible to reduce the sequential running time from $O\left(N \log ^{2} N\right)$ to $O(N \log N)$ ? Is it possible to reduce the circuit depth of the parallel algorithm from $O\left(\log ^{2} N\right)$ to $O(\log N)$, without increasing the circuit size beyond polynomial? Is it possible to reduce the circuit size from $O\left(N^{2}\right)$ to $O(N \log N)$, while maintaining poly-logarithmic circuit depth? More generally, can the complexity of the generic algorithm (for arbitrary $p$ ) be improved from $O\left(N \log ^{2} N / \sqrt{p}\right)$ to $O(N \log N / p)$ ?

On a different front, is it possible to efficiently decode Barnes-Wall lattices beyond the squared unique decoding radius? Can the maximum-likelihood decoding problem (i.e., the closest vector problem) be solved in polynomial time? Is it possible to list decode $\mathrm{BW}^{n}$, and up to what radius?

\section{Acknowledgments}

The authors thank Alex Vardy, Ilya Dumer and the anonymous referees for interesting conversations and pointers to the literature. The first author was supported in part by NSF grant CCF-0634909. Any opinions, findings, and conclusions or recommendations expressed in this material are those of the author(s) and do not necessarily reflect the views of the National Science Foundation. 


\section{References}

[1] D. Agrawal and A. Vardy. Generalized minimum-distance decoding in Euclidean space: performance analysis. IEEE Transactions on Information Theory, 46(1):60-83, 2000.

[2] A. Banihashemi and I. Blake. Trellis complexity and minimal trellis diagrams of lattices. IEEE Transactions on Information Theory, 44(5):1829-1847, 1998.

[3] G.D. Forney. Coset codes. II. Binary lattices and related codes. IEEE Transactions on Information Theory, 34(5):1152-1187, 1988.

[4] G.D. Forney and A. Vardy. Generalized minimum-distance decoding of Euclidean-space codes and lattices. IEEE Transactions on Information Theory, 42(6):1992-2026, 1996.

[5] G. Kabatyanskii. On decoding of Reed-Muller codes in semicontinuous channels. In Intl. Workshop on Algebra and Combinatorial Coding Theory, pages 87-91, 1990.

[6] G. Nebe, E. Rains, and N. Sloane. The invariants of the Clifford groups. Designs, Codes and Cryptography, 24(1):99-122, 2001.

[7] G. Nebe, E. Rains, and N. Sloane. A simple construction for the Barnes-Wall lattices. Presented at the Forney Festschrift at MIT, based on [6], 2002.

[8] M. Ran and J. Snyders. Efficient decoding of the Gosset, Coxeter-Todd and the Barnes-Wall lattices. In IEEE International Symposium on Information Theory, page 92, 1998.

[9] A. Salomon and O. Amrani. Augmented product codes and lattices: Reed-Muller codes and Barnes-Wall lattices. IEEE Transactions on Information Theory, 51(11):3918-3930, 2005.

[10] G. Schnabl and M. Bossert. Soft-decision decoding of Reed-Muller codes as generalized multiple concatenated codes. IEEE Transactions on Information Theory, 41(1):304-308, 1995.

[11] K. Wahlgren and Z.-X. Wan. Iterated squaring construction of bi-infinite group partition chain. In IEEE International Symposium on Information Theory, page 442, 1997.

[12] C. Wang, B. Shen, and K. Tzeng. Generalised minimum distance decoding of Reed-Muller codes and Barnes-Wall lattices. In IEEE International Symposium on Information Theory, page 186, 1995. 